\title{
Shark: SQL and Rich Analytics at Scale
}

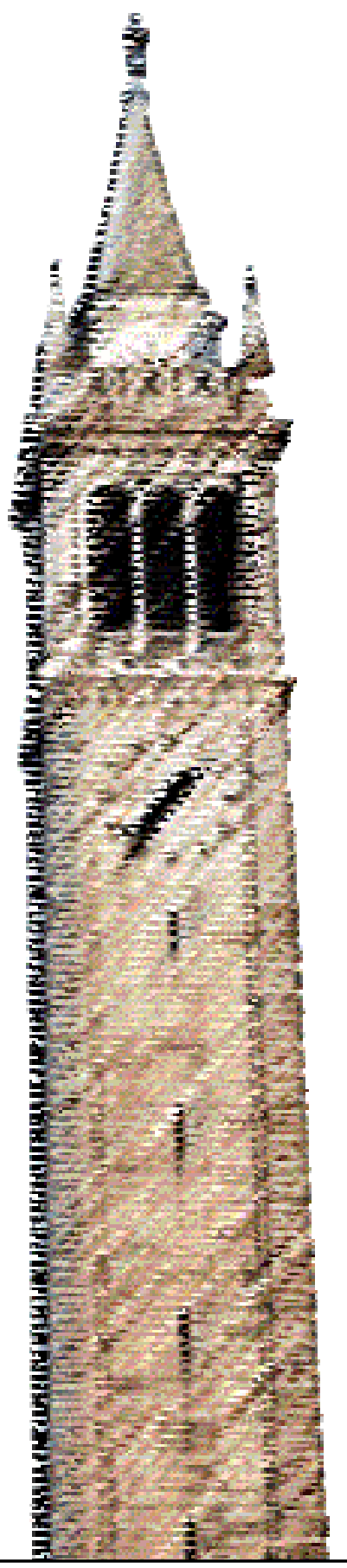

Reynold Shi Xin

Joshua Rosen

Matei Zaharia

Michael Franklin

Scott Shenker

Ion Stoica

Electrical Engineering and Computer Sciences University of California at Berkeley

Technical Report No. UCB/EECS-2012-214

http://www.eecs.berkeley.edu/Pubs/TechRpts/2012/EECS-2012-214.html

November 26, 2012 


\section{Report Documentation Page}

Form Approved

OMB No. 0704-0188

Public reporting burden for the collection of information is estimated to average 1 hour per response, including the time for reviewing instructions, searching existing data sources, gathering and maintaining the data needed, and completing and reviewing the collection of information. Send comments regarding this burden estimate or any other aspect of this collection of information,

including suggestions for reducing this burden, to Washington Headquarters Services, Directorate for Information Operations and Reports, 1215 Jefferson Davis Highway, Suite 1204, Arlington

VA 22202-4302. Respondents should be aware that notwithstanding any other provision of law, no person shall be subject to a penalty for failing to comply with a collection of information if it

does not display a currently valid OMB control number.

\begin{tabular}{|c|c|c|}
\hline $\begin{array}{l}\text { 1. REPORT DATE } \\
\mathbf{2 6} \text { NOV } \mathbf{2 0 1 2}\end{array}$ & 2. REPORT TYPE & $\begin{array}{l}\text { 3. DATES COVERED } \\
\mathbf{0 0 - 0 0 - 2 0 1 2} \text { to 00-00-2012 }\end{array}$ \\
\hline \multirow{3}{*}{\multicolumn{2}{|c|}{$\begin{array}{l}\text { 4. TITLE AND SUBTITLE } \\
\text { Shark: SQL and Rich Analytics at Scale }\end{array}$}} & 5a. CONTRACT NUMBER \\
\hline & & 5b. GRANT NUMBER \\
\hline & & 5c. PROGRAM ELEMENT NUMBER \\
\hline \multirow{3}{*}{\multicolumn{2}{|c|}{ 6. AUTHOR(S) }} & 5d. PROJECT NUMBER \\
\hline & & 5e. TASK NUMBER \\
\hline & & 5f. WORK UNIT NUMBER \\
\hline \multicolumn{2}{|c|}{$\begin{array}{l}\text { 7. PERFORMING ORGANIZATION NAME(S) AND ADDRESS(ES) } \\
\text { University of California at Berkeley,Electrical Engineering and } \\
\text { Computer Sciences,Berkeley,CA,94720 }\end{array}$} & $\begin{array}{l}\text { 8. PERFORMING ORGANIZATION } \\
\text { REPORT NUMBER }\end{array}$ \\
\hline \multirow{2}{*}{\multicolumn{2}{|c|}{ 9. SPONSORING/MONITORING AGENCY NAME(S) AND ADDRESS(ES) }} & 10. SPONSOR/MONITOR'S ACRONYM(S) \\
\hline & & $\begin{array}{l}\text { 11. SPONSOR/MONITOR'S REPORT } \\
\text { NUMBER(S) }\end{array}$ \\
\hline
\end{tabular}

12. DISTRIBUTION/AVAILABILITY STATEMENT

Approved for public release; distribution unlimited

13. SUPPLEMENTARY NOTES

14. ABSTRACT

Shark is a new data analysis system that marries query processing with complex analytics on large clusters. It leverages a novel distributed memory abstraction to provide a unified engine that can run SQL queries and sophisticated analytics functions (e.g., iterative machine learning) at scale, and efficiently recovers from failures mid-query. This allows Shark to run SQL queries up to 100 faster than Apache Hive, and machine learning programs up to 100 faster than Hadoop. Unlike previous systems, Shark shows that it is possible to achieve these speedups while retaining a MapReduce-like execution engine, and the fine-grained fault tolerance properties that such engines provide. It extends such an engine in several ways, including column-oriented in-memory storage and dynamic mid-query replanning, to effectively execute SQL. The result is a system that matches the speedups reported for MPP analytic databases over MapReduce, while offering fault tolerance properties and complex analytics capabilities that they lack.

\begin{tabular}{|c|c|c|c|c|c|}
\hline \multicolumn{3}{|c|}{ 16. SECURITY CLASSIFICATION OF: } & \multirow{2}{*}{$\begin{array}{l}\text { 17. LIMITATION OF } \\
\text { ABSTRACT } \\
\text { Same as } \\
\text { Report (SAR) }\end{array}$} & \multirow{2}{*}{$\begin{array}{c}\text { 18. NUMBER } \\
\text { OF PAGES } \\
\mathbf{1 4}\end{array}$} & \multirow{2}{*}{$\begin{array}{l}\text { 19a. NAME OF } \\
\text { RESPONSIBLE PERSON }\end{array}$} \\
\hline $\begin{array}{l}\text { a. REPORT } \\
\text { unclassified }\end{array}$ & $\begin{array}{l}\text { b. ABSTRACT } \\
\text { unclassified }\end{array}$ & $\begin{array}{l}\text { c. THIS PAGE } \\
\text { unclassified }\end{array}$ & & & \\
\hline
\end{tabular}


Copyright (C) 2012, by the author(s).

All rights reserved.

Permission to make digital or hard copies of all or part of this work for personal or classroom use is granted without fee provided that copies are not made or distributed for profit or commercial advantage and that copies bear this notice and the full citation on the first page. To copy otherwise, to republish, to post on servers or to redistribute to lists, requires prior specific permission. 


\title{
Shark: SQL and Rich Analytics at Scale
}

\author{
Reynold Xin, Josh Rosen, Matei Zaharia, \\ Michael J. Franklin, Scott Shenker, Ion Stoica \\ AMPLab, EECS, UC Berkeley \\ \{rxin, joshrosen, matei, franklin, shenker, istoica\}@cs.berkeley.edu
}

\begin{abstract}
Shark is a new data analysis system that marries query processing with complex analytics on large clusters. It leverages a novel distributed memory abstraction to provide a unified engine that can run SQL queries and sophisticated analytics functions (e.g., iterative machine learning) at scale, and efficiently recovers from failures mid-query. This allows Shark to run SQL queries up to $100 \times$ faster than Apache Hive, and machine learning programs up to $100 \times$ faster than Hadoop. Unlike previous systems, Shark shows that it is possible to achieve these speedups while retaining a MapReduce-like execution engine, and the fine-grained fault tolerance properties that such engines provide. It extends such an engine in several ways, including column-oriented in-memory storage and dynamic mid-query replanning, to effectively execute SQL. The result is a system that matches the speedups reported for MPP analytic databases over MapReduce, while offering fault tolerance properties and complex analytics capabilities that they lack.
\end{abstract}

\section{Introduction}

Modern data analysis faces a confluence of growing challenges. First, data volumes are expanding dramatically, creating the need to scale out across clusters of hundreds of commodity machines. Second, this new scale increases the incidence of faults and stragglers (slow tasks), complicating parallel database design. Third, the complexity of data analysis has also grown: modern data analysis employs sophisticated statistical methods, such as machine learning algorithms, that go well beyond the roll-up and drill-down capabilities of traditional enterprise data warehouse systems. Finally, despite these increases in scale and complexity, users still expect to be able to query data at interactive speeds.

To tackle the "big data" problem, two major lines of systems have recently been explored. The first, composed of MapReduce [13] and various generalizations [17, 9], offers a fine-grained fault tolerance model suitable for large clusters, where tasks on failed or slow nodes can be deterministically re-executed on other nodes. MapReduce is also fairly general: it has been shown to be able to express many statistical and learning algorithms [11]. It also easily supports unstructured data and "schema-on-read." However, MapReduce engines lack many of the features that make databases efficient, and have high latencies of tens of seconds to hours. Even systems that have significantly optimized MapReduce for SQL queries, such as Google's Tenzing [9], or that combine it with a traditional database on each node, such as HadoopDB [3], report a minimum latency of 10 seconds. As such, MapReduce approaches have largely been dismissed for interactive-speed queries [25], and even Google is developing new engines for such workloads [24].

Instead, most MPP analytic databases (e.g., Vertica, Greenplum, Teradata) and several of the new low-latency engines proposed for
MapReduce environments (e.g., Google Dremel [24], Cloudera Impala [1]) employ a coarser-grained recovery model, where an entire query has to be resubmitted if a machine fails. ${ }^{1}$ This works well for short queries where a retry is inexpensive, but faces significant challenges in long queries as clusters scale up [3]. In addition, these systems often lack the rich analytics functions that are easy to implement in MapReduce, such as machine learning and graph algorithms. Furthermore, while it may be possible to implement some of these functions using UDFs, these algorithms are often expensive, furthering the need for fault and straggler recovery for long queries. Thus, most organizations tend to use other systems alongside MPP databases to perform complex analytics.

To provide an effective environment for big data analysis, we believe that processing systems will need to support both SQL and complex analytics efficiently, and to provide fine-grained fault recovery across both types of operations. This paper describes a new system that meets these goals, called Shark. Shark is open source and compatible with Apache Hive, and has already been used at web companies to speed up queries by $40-100 \times$.

Shark builds on a recently-proposed distributed shared memory abstraction called Resilient Distributed Datasets (RDDs) [33] to perform most computations in memory while offering fine-grained fault tolerance. In-memory computing is increasingly important in large-scale analytics for two reasons. First, many complex analytics functions, such as machine learning and graph algorithms, are iterative, going over the data multiple times; thus, the fastest systems deployed for these applications are in-memory [23, 22, 33]. Second, even traditional SQL warehouse workloads exhibit strong temporal and spatial locality, because more-recent fact table data and small dimension tables are read disproportionately often. A study of Facebook's Hive warehouse and Microsoft's Bing analytics cluster showed that over $95 \%$ of queries in both systems could be served out of memory using just $64 \mathrm{~GB}$ /node as a cache, even though each system manages more than $100 \mathrm{~PB}$ of total data [5].

The main benefit of RDDs is an efficient mechanism for fault recovery. Traditional main-memory databases support fine-grained updates to tables and replicate writes across the network for fault tolerance, which is expensive on large commodity clusters. In contrast, RDDs restrict the programming interface to coarse-grained deterministic operators that affect multiple data items at once, such as map, group-by and join, and recover from failures by tracking the lineage of each dataset and recomputing lost data. This approach works well for data-parallel relational queries, and has also been shown to support machine learning and graph computation [33]. Thus, when a node fails, Shark can recover mid-query by rerun-

\footnotetext{
${ }^{1}$ Dremel provides fault tolerance within a query, but Dremel is limited to aggregation trees instead of the more complex communication patterns in joins.
} 


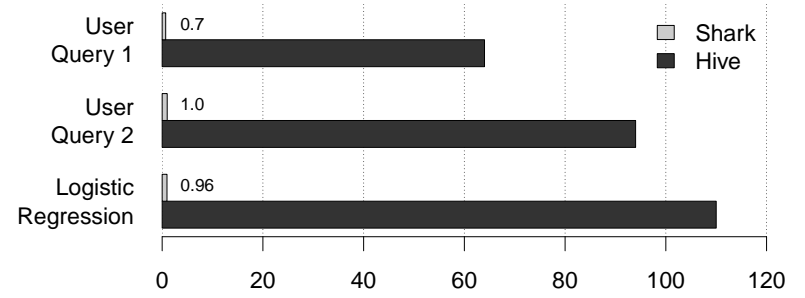

Figure 1: Performance of Shark vs. Hive/Hadoop on two SQL queries from an early user and one iteration of logistic regression (a classification algorithm that runs $\sim 10$ such steps). Results measure the runtime (seconds) on a 100-node cluster.

ning the deterministic operations used to build lost data partitions on other nodes, similar to MapReduce. Indeed, it typically recovers within seconds, by parallelizing this work across the cluster.

To run SQL efficiently, however, we also had to extend the RDD execution model, bringing in several concepts from traditional analytical databases and some new ones. We started with an existing implementation of RDDs called Spark [33], and added several features. First, to store and process relational data efficiently, we implemented in-memory columnar storage and columnar compression. This reduced both the data size and the processing time by as much as $5 \times$ over naïvely storing the data in a Spark program in its original format. Second, to optimize SQL queries based on the data characteristics even in the presence of analytics functions and UDFs, we extended Spark with Partial DAG Execution (PDE): Shark can reoptimize a running query after running the first few stages of its task DAG, choosing better join strategies or the right degree of parallelism based on observed statistics. Third, we leverage other properties of the Spark engine not present in traditional MapReduce systems, such as control over data partitioning.

Our implementation of Shark is compatible with Apache Hive [28], supporting all of Hive's SQL dialect and UDFs and allowing execution over unmodified Hive data warehouses. It augments SQL with complex analytics functions written in Spark, using Spark's Java, Scala or Python APIs. These functions can be combined with SQL in a single execution plan, providing in-memory data sharing and fast recovery across both types of processing.

Experiments show that using RDDs and the optimizations above, Shark can answer SQL queries up to $100 \times$ faster than Hive, runs iterative machine learning algorithms up to $100 \times$ faster than Hadoop, and can recover from failures mid-query within seconds. Figure 1 shows three sample results. Shark's speed is comparable to that of MPP databases in benchmarks like Pavlo et al.'s comparison with MapReduce [25], but it offers fine-grained recovery and complex analytics features that these systems lack.

More fundamentally, our work shows that MapReduce-like execution models can be applied effectively to SQL, and offer a promising way to combine relational and complex analytics. In the course of presenting of Shark, we also explore why SQL engines over previous MapReduce runtimes, such as Hive, are slow, and show how a combination of enhancements in Shark (e.g., PDE), and engine properties that have not been optimized in MapReduce, such as the overhead of launching tasks, eliminate many of the bottlenecks in traditional MapReduce systems.

\section{System Overview}

Shark is a data analysis system that supports both SQL query processing and machine learning functions. We have chosen to imple-
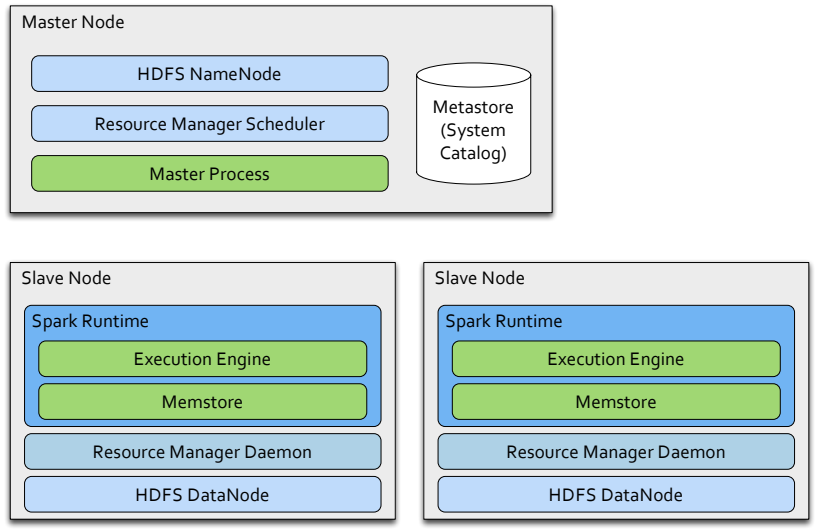

Figure 2: Shark Architecture

ment Shark to be compatible with Apache Hive. It can be used to query an existing Hive warehouse and return results much faster, without modification to either the data or the queries.

Thanks to its Hive compatibility, Shark can query data in any system that supports the Hadoop storage API, including HDFS and Amazon S3. It also supports a wide range of data formats such as text, binary sequence files, JSON, and XML. It inherits Hive's schema-on-read capability and nested data types [28].

In addition, users can choose to load high-value data into Shark's memory store for fast analytics, as shown below:

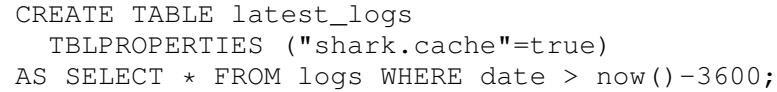

Figure 2 shows the architecture of a Shark cluster, consisting of a single master node and a number of slave nodes, with the warehouse metadata stored in an external transactional database. It is built on top of Spark, a modern MapReduce-like cluster computing engine. When a query is submitted to the master, Shark compiles the query into operator tree represented as RDDs, as we shall discuss in Section 2.4. These RDDs are then translated by Spark into a graph of tasks to execute on the slave nodes.

Cluster resources can optionally be allocated by a cluster resource manager (e.g., Hadoop YARN or Apache Mesos) that provides resource sharing and isolation between different computing frameworks, allowing Shark to coexist with engines like Hadoop.

In the remainder of this section, we cover the basics of Spark and the RDD programming model, followed by an explanation of how Shark query plans are generated and run.

\subsection{Spark}

Spark is the MapReduce-like cluster computing engine used by Shark. Spark has several features that differentiate it from traditional MapReduce engines [33]:

1. Like Dryad and Tenzing [17, 9], it supports general computation DAGs, not just the two-stage MapReduce topology.

2. It provides an in-memory storage abstraction called Resilient Distributed Datasets (RDDs) that lets applications keep data in memory across queries, and automatically reconstructs it after failures [33].

3. The engine is optimized for low latency. It can efficiently manage tasks as short as 100 milliseconds on clusters of thousands of cores, while engines like Hadoop incur a latency of 5-10 seconds to launch each task. 


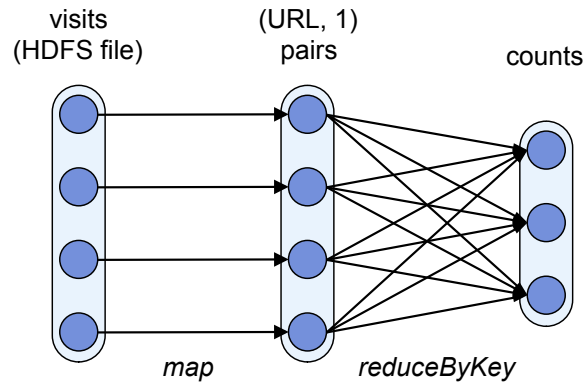

Figure 3: Lineage graph for the RDDs in our Spark example. Oblongs represent RDDs, while circles show partitions within a dataset. Lineage is tracked at the granularity of partitions.

RDDs are unique to Spark, and were essential to enabling midquery fault tolerance. However, the other differences are important engineering elements that contribute to Shark's performance.

On top of these features, we have also modified the Spark engine for Shark to support partial DAG execution, that is, modification of the query plan DAG after only some of the stages have finished, based on statistics collected from these stages. Similar to [20], we use this technique to optimize join algorithms and other aspects of the execution mid-query, as we shall discuss in Section 3.1.

\subsection{Resilient Distributed Datasets (RDDs)}

Spark's main abstraction is resilient distributed datasets (RDDs), which are immutable, partitioned collections that can be created through various data-parallel operators (e.g., map, group-by, hashjoin). Each RDD is either a collection stored in an external storage system, such as a file in HDFS, or a derived dataset created by applying operators to other RDDs. For example, given an RDD of (visitID, URL) pairs for visits to a website, we might compute an RDD of (URL, count) pairs by applying a map operator to turn each event into an (URL, 1) pair, and then a reduce to add the counts by URL.

In Spark's native API, RDD operations are invoked through a functional interface similar to DryadLINQ [19] in Scala, Java or Python. For example, the Scala code for the query above is:

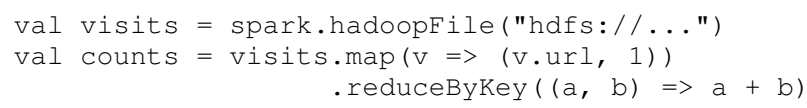

RDDs can contain arbitrary data types as elements (since Spark runs on the JVM, these elements are Java objects), and are automatically partitioned across the cluster, but they are immutable once created, and they can only be created through Spark's deterministic parallel operators. These two restrictions, however, enable highly efficient fault recovery. In particular, instead of replicating each RDD across nodes for fault-tolerance, Spark remembers the lineage of the RDD (the graph of operators used to build it), and recovers lost partitions by recomputing them from base data [33]. ${ }^{2}$ For example, Figure 3 shows the lineage graph for the RDDs computed above. If Spark loses one of the partitions in the (URL, 1) $\mathrm{RDD}$, for example, it can recompute it by rerunning the map on just the corresponding partition of the input file.

The RDD model offers several key benefits our large-scale inmemory computing setting. First, RDDs can be written at the speed of DRAM instead of the speed of the network, because there is no

\footnotetext{
${ }^{2}$ We assume that external files for RDDs representing external data do not change, or that we can take a snapshot of a file when we create an RDD from it.
}

need to replicate each byte written to another machine for faulttolerance. DRAM in a modern server is over $10 \times$ faster than even a 10-Gigabit network. Second, Spark can keep just one copy of each RDD partition in memory, saving precious memory over a replicated system, since it can always recover lost data using lineage. Third, when a node fails, its lost RDD partitions can be rebuilt in parallel across the other nodes, allowing speedy recovery. ${ }^{3}$ Fourth, even if a node is just slow (a "straggler"), we can recompute necessary partitions on other nodes because RDDs are immutable so there are no consistency concerns with having two copies of a partition. These benefits make RDDs attractive as the foundation for our relational processing in Shark.

\subsection{Fault Tolerance Guarantees}

To summarize the benefits of RDDs explained above, Shark provides the following fault tolerance properties, which have been difficult to support in traditional MPP database designs:

1. Shark can tolerate the loss of any set of worker nodes. The execution engine will re-execute any lost tasks and recompute any lost RDD partitions using lineage. ${ }^{4}$ This is true even within a query: Spark will rerun any failed tasks, or lost dependencies of new tasks, without aborting the query.

2. Recovery is parallelized across the cluster. If a failed node contained 100 RDD partitions, these can be rebuilt in parallel on 100 different nodes, quickly recovering the lost data.

3. The deterministic nature of RDDs also enables straggler mitigation: if a task is slow, the system can launch a speculative "backup copy" of it on another node, as in MapReduce [13].

4. Recovery works even in queries that combine SQL and machine learning UDFs (Section 4), as these operations all compile into a single RDD lineage graph.

\subsection{Executing SQL over RDDs}

Shark runs SQL queries over Spark using a three-step process similar to traditional RDBMSs: query parsing, logical plan generation, and physical plan generation.

Given a query, Shark uses the Hive query compiler to parse the query and generate an abstract syntax tree. The tree is then turned into a logical plan and basic logical optimization, such as predicate pushdown, is applied. Up to this point, Shark and Hive share an identical approach. Hive would then convert the operator into a physical plan consisting of multiple MapReduce stages. In the case of Shark, its optimizer applies additional rule-based optimizations, such as pushing LIMIT down to individual partitions, and creates a physical plan consisting of transformations on RDDs rather than MapReduce jobs. We use a variety of operators already present in Spark, such as map and reduce, as well as new operators we implemented for Shark, such as broadcast joins. Spark's master then executes this graph using standard MapReduce scheduling techniques, such placing tasks close to their input data, rerunning lost tasks, and performing straggler mitigation [33].

While this basic approach makes it possible to run SQL over Spark, doing so efficiently is challenging. The prevalence of UDFs and complex analytic functions in Shark's workload makes it difficult to determine an optimal query plan at compile time, especially for new data that has not undergone ETL. In addition, even with

\footnotetext{
${ }^{3}$ To provide fault tolerance across "shuffle" operations like a parallel reduce, the execution engine also saves the "map" side of the shuffle in memory on the source nodes, spilling to disk if necessary. ${ }^{4}$ Support for master recovery could also be added by reliabliy logging the RDD lineage graph and the submitted jobs, because this state is small, but we have not yet implemented this.
} 
such a plan, naïvely executing it over Spark (or other MapReduce runtimes) can be inefficient. In the next section, we discuss several extensions we made to Spark to efficiently store relational data and run SQL, starting with a mechanism that allows for dynamic, statistics-driven re-optimization at run-time.

\section{Engine Extensions}

In this section, we describe our modifications to the Spark engine to enable efficient execution of SQL queries.

\subsection{Partial DAG Execution (PDE)}

Systems like Shark and Hive are frequently used to query fresh data that has not undergone a data loading process. This precludes the use of static query optimization techniques that rely on accurate a priori data statistics, such as statistics maintained by indices. The lack of statistics for fresh data, combined with the prevalent use of UDFs, necessitates dynamic approaches to query optimization.

To support dynamic query optimization in a distributed setting, we extended Spark to support partial DAG execution (PDE), a technique that allows dynamic alteration of query plans based on data statistics collected at run-time.

We currently apply partial DAG execution at blocking "shuffle" operator boundaries where data is exchanged and repartitioned, since these are typically the most expensive operations in Shark. By default, Spark materializes the output of each map task in memory before a shuffle, spilling it to disk as necessary. Later, reduce tasks fetch this output.

PDE modifies this mechanism in two ways. First, it gathers customizable statistics at global and per-partition granularities while materializing map output. Second, it allows the DAG to be altered based on these statistics, either by choosing different operators or altering their parameters (such as their degrees of parallelism).

These statistics are customizable using a simple, pluggable accumulator API. Some example statistics include:

1. Partition sizes and record counts, which can be used to detect skew.

2. Lists of "heavy hitters," i.e., items that occur frequently in the dataset.

3. Approximate histograms, which can be used to estimate partitions' data's distributions.

These statistics are sent by each worker to the master, where they are aggregated and presented to the optimizer. For efficiency, we use lossy compression to record the statistics, limiting their size to 1-2 KB per task. For instance, we encode partition sizes (in bytes) with logarithmic encoding, which can represent sizes of up to 32 GB using only one byte with at most $10 \%$ error. The master can then use these statistics to perform various run-time optimizations, as we shall discuss next.

Partial DAG execution complements existing adaptive query optimization techniques that typically run in a single-node system [6, 20,30 ], as we can use existing techniques to dynamically optimize the local plan within each node, and use PDE to optimize the global structure of the plan at stage boundaries. This fine-grained statistics collection, and the optimizations that it enables, differentiates PDE from graph rewriting features in previous systems, such as DryadLINQ [19].

\subsubsection{Join Optimization}

Partial DAG execution can be used to perform several run-time optimizations for join queries.

Figure 4 illustrates two communication patterns for MapReducestyle joins. In shuffle join, both join tables are hash-partitioned by

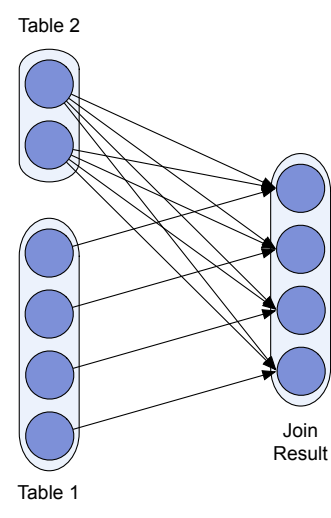

Map join

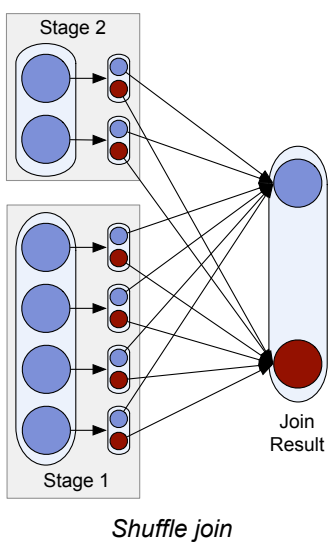

Figure 4: Data flows for map join and shuffle join. Map join broadcasts the small table to all large table partitions, while shuffle join repartitions and shuffles both tables.

the join key. Each reducer joins corresponding partitions using a local join algorithm, which is chosen by each reducer based on runtime statistics. If one of a reducer's input partitions is small, then it constructs a hash table over the small partition and probes it using the large partition. If both partitions are large, then a symmetric hash join is performed by constructing hash tables over both inputs.

In map join, also known as broadcast join, a small input table is broadcast to all nodes, where it is joined with each partition of a large table. This approach can result in significant cost savings by avoiding an expensive repartitioning and shuffling phase.

Map join is only worthwhile if some join inputs are small, so Shark uses partial DAG execution to select the join strategy at runtime based on its inputs' exact sizes. By using sizes of the join inputs gathered at run-time, this approach works well even with input tables that have no prior statistics, such as intermediate results.

Run-time statistics also inform the join tasks' scheduling policies. If the optimizer has a prior belief that a particular join input will be small, it will schedule that task before other join inputs and decide to perform a map-join if it observes that the task's output is small. This allows the query engine to avoid performing the preshuffle partitioning of a large table once the optimizer has decided to perform a map-join.

\subsubsection{Skew-handling and Degree of Parallelism}

Partial DAG execution can also be used to determine operators' degrees of parallelism and to mitigate skew.

The degree of parallelism for reduce tasks can have a large performance impact: launching too few reducers may overload reducers' network connections and exhaust their memories, while launching too many may prolong the job due to task scheduling overhead. Hive's performance is especially sensitive to the number of reduce tasks, due to Hadoop's large scheduling overhead.

Using partial DAG execution, Shark can use individual partitions' sizes to determine the number of reducers at run-time by coalescing many small, fine-grained partitions into fewer coarse partitions that are used by reduce tasks. To mitigate skew, fine-grained partitions are assigned to coalesced partitions using a greedy binpacking heuristic that attempts to equalize coalesced partitions' sizes [15]. This offers performance benefits, especially when good bin-packings exist.

Somewhat surprisingly, we discovered that Shark can obtain similar performance improvement by running a larger number of reduce tasks. We attribute this to Spark's low scheduling overhead. 


\subsection{Columnar Memory Store}

In-memory computation is essential to low-latency query answering, given that memory's throughput is orders of magnitude higher than that of disks. Naïvely using Spark's memory store, however, can lead to undesirable performance. Shark implements a columnar memory store on top of Spark's memory store.

In-memory data representation affects both space footprint and read throughput. A naïve approach is to simply cache the on-disk data in its native format, performing on-demand deserialization in the query processor. This deserialization becomes a major bottleneck: in our studies, we saw that modern commodity CPUs can deserialize at a rate of only $200 \mathrm{MB}$ per second per core.

The approach taken by Spark's default memory store is to store data partitions as collections of JVM objects. This avoids deserialization, since the query processor can directly use these objects, but leads to significant storage space overheads. Common JVM implementations add 12 to 16 bytes of overhead per object. For example, storing $270 \mathrm{MB}$ of TPC-H lineitem table as JVM objects uses approximately $971 \mathrm{MB}$ of memory, while a serialized representation requires only $289 \mathrm{MB}$, nearly three times less space. A more serious implication, however, is the effect on garbage collection (GC). With a 200 B record size, a 32 GB heap can contain 160 million objects. The JVM garbage collection time correlates linearly with the number of objects in the heap, so it could take minutes to perform a full GC on a large heap. These unpredictable, expensive garbage collections cause large variability in workers' response times.

Shark stores all columns of primitive types as JVM primitive arrays. Complex data types supported by Hive, such as map and array, are serialized and concatenated into a single byte array. Each column creates only one JVM object, leading to fast GCs and a compact data representation. The space footprint of columnar data can be further reduced by cheap compression techniques at virtually no CPU cost. Similar to more traditional database systems [27], Shark implements CPU-efficient compression schemes such as dictionary encoding, run-length encoding, and bit packing.

Columnar data representation also leads to better cache behavior, especially for for analytical queries that frequently compute aggregations on certain columns.

\subsection{Distributed Data Loading}

In addition to query execution, Shark also uses Spark's execution engine for distributed data loading. During loading, a table is split into small partitions, each of which is loaded by a Spark task. The loading tasks use the data schema to extract individual fields from rows, marshals a partition of data into its columnar representation, and stores those columns in memory.

Each data loading task tracks metadata to decide whether each column in a partition should be compressed. For example, the loading task will compress a column using dictionary encoding if its number of distinct values is below a threshold. This allows each task to choose the best compression scheme for each partition, rather than conforming to a global compression scheme that might not be optimal for local partitions. These local decisions do not require coordination among data loading tasks, allowing the load phase to achieve a maximum degree of parallelism, at the small cost of requiring each partition to maintain its own compression metadata. It is important to clarify that an RDD's lineage does not need to contain the compression scheme and metadata for each partition. The compression scheme and metadata are simply byproducts of the RDD computation, and can be deterministically recomputed along with the in-memory data in the case of failures.

As a result, Shark can load data into memory at the aggregated throughput of the CPUs processing incoming data.
Pavlo et al.[25] showed that Hadoop was able to perform data loading at 5 to 10 times the throughput of MPP databases. Tested using the same dataset used in [25], Shark provides the same throughput as Hadoop in loading data into HDFS. Shark is 5 times faster than Hadoop when loading data into its memory store.

\subsection{Data Co-partitioning}

In some warehouse workloads, two tables are frequently joined together. For example, the TPC-H benchmark frequently joins the lineitem and order tables. A technique commonly used by MPP databases is to co-partition the two tables based on their join key in the data loading process. In distributed file systems like HDFS, the storage system is schema-agnostic, which prevents data copartitioning. Shark allows co-partitioning two tables on a common key for faster joins in subsequent queries. This can be accomplished with the DISTRIBUTE BY clause:

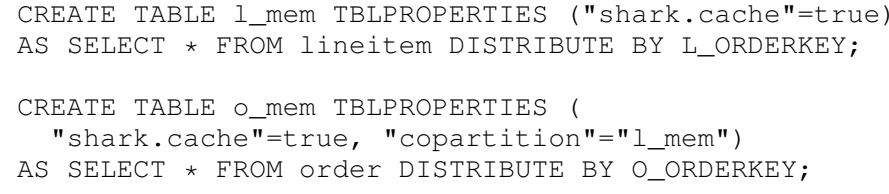

When joining two co-partitioned tables, Shark's optimizer constructs a DAG that avoids the expensive shuffle and instead uses map tasks to perform the join.

\subsection{Partition Statistics and Map Pruning}

Data tend to be stored in some logical clustering on one or more columns. For example, entries in a website's traffic log data might be grouped by users' physical locations, because logs are first stored in data centers that have the best geographical proximity to users. Within each data center, logs are append-only and are stored in roughly chronological order. As a less obvious case, a news site's logs might contain news_id and timestamp columns that have strongly correlated values. For analytical queries, it is typical to apply filter predicates or aggregations over such columns. For example, a daily warehouse report might describe how different visitor segments interact with the website; this type of query naturally applies a predicate on timestamps and performs aggregations that are grouped by geographical location. This pattern is even more frequent for interactive data analysis, during which drill-down operations are frequently performed.

Map pruning is the process of pruning data partitions based on their natural clustering columns. Since Shark's memory store splits data into small partitions, each block contains only one or few logical groups on such columns, and Shark can avoid scanning certain blocks of data if their values fall out of the query's filter range.

To take advantage of these natural clusterings of columns, Shark's memory store on each worker piggybacks the data loading process to collect statistics. The information collected for each partition include the range of each column and the distinct values if the number of distinct values is small (i.e., enum columns). The collected statistics are sent back to the master program and kept in memory for pruning partitions during query execution.

When a query is issued, Shark evaluates the query's predicates against all partition statistics; partitions that do not satisfy the predicate are pruned and Shark does not launch tasks to scan them.

We collected a sample of queries from the Hive warehouse of a video analytics company, and out of the 3833 queries we obtained, at least 3277 of them contain predicates that Shark can use for map pruning. Section 6 provides more details on this workload. 


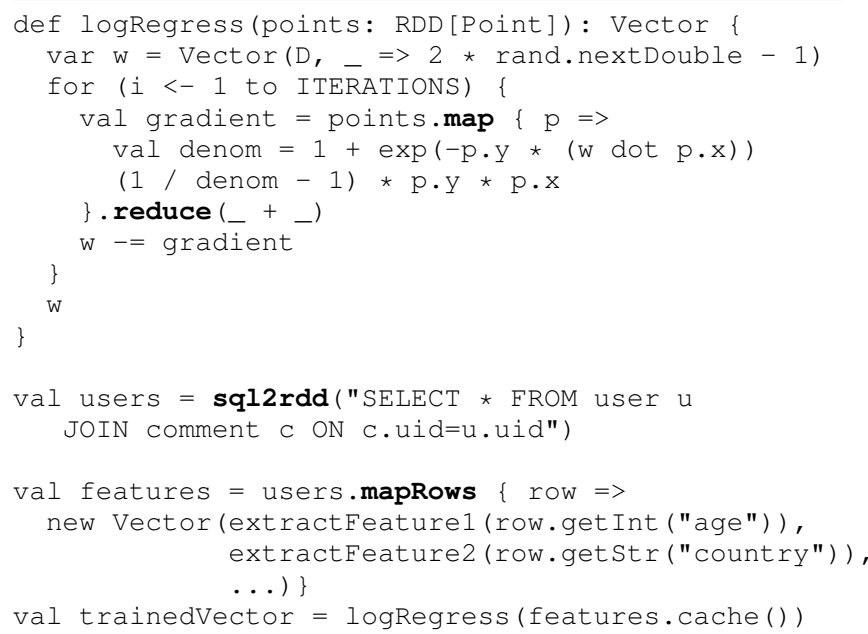

\section{Listing 1: Logistic Regression Example}

\section{Machine Learning Support}

A key design goal of Shark is to provide a single system capable of efficient SQL query processing and sophisticated machine learning. Following the principle of pushing computation to data, Shark supports machine learning as a first-class citizen. This is enabled by the design decision to choose Spark as the execution engine and RDD as the main data structure for operators. In this section, we explain Shark's language and execution engine integration for SQL and machine learning.

Other research projects $[12,14]$ have demonstrated that it is possible to express certain machine learning algorithms in SQL and avoid moving data out of the database. The implementation of those projects, however, involves a combination of SQL, UDFs, and driver programs written in other languages. The systems become obscure and difficult to maintain; in addition, they may sacrifice performance by performing expensive parallel numerical computations on traditional database engines that were not designed for such workloads. Contrast this with the approach taken by Shark, which offers in-database analytics that push computation to data, but does so using a runtime that is optimized for such workloads and a programming model that is designed to express machine learning algorithms.

\subsection{Language Integration}

In addition to executing a SQL query and returning its results, Shark also allows queries to return the RDD representing the query plan. Callers to Shark can then invoke distributed computation over the query result using the returned RDD.

As an example of this integration, Listing 1 illustrates a data analysis pipeline that performs logistic regression over a user database. Logistic regression, a common classification algorithm, searches for a hyperplane $w$ that best separates two sets of points (e.g. spammers and non-spammers). The algorithm applies gradient descent optimization by starting with a randomized $w$ vector and iteratively updating it by moving along gradients towards an optimum value.

The program begins by using sql2rdd to issue a SQL query to retreive user information as a TableRDD. It then performs feature extraction on the query rows and runs logistic regression over the extracted feature matrix. Each iteration of logRegress applies a function of $w$ to all data points to produce a set of gradients, which are summed to produce a net gradient that is used to update $w$.
The highlighted map, mapRows, and reduce functions are automatically parallelized by Shark to execute across a cluster, and the master program simply collects the output of the reduce function to update $w$.

Note that this distributed logistic regression implementation in Shark looks remarkably similar to a program implemented for a single node in the Scala language. The user can conveniently mix the best parts of both SQL and MapReduce-style programming.

Currently, Shark provides native support for Scala and Java, with support for Python in development. We have modified the Scala shell to enable interactive execution of both SQL and distributed machine learning algorithms. Because Shark is built on top of the JVM, it is trivial to support other JVM languages, such as Clojure or JRuby.

We have implemented a number of basic machine learning algorithms, including linear regression, logistic regression, and kmeans clustering. In most cases, the user only needs to supply a mapRows function to perform feature extraction and can invoke the provided algorithms.

The above example demonstrates how machine learning computations can be performed on query results. Using RDD as the main data structure for query operators also enables the possibility of using SQL to query the results of machine learning computations in a single execution plan.

\subsection{Execution Engine Integration}

In addition to language integration, another key benefit of using RDDs as the data structure for operators is the execution engine integration. This common abstraction allows machine learning computations and SQL queries to share workers and cached data without the overhead of data movement.

Because SQL query processing is implemented using RDDs, lineage is kept for the whole pipeline, which enables end-to-end fault tolerance for the entire workflow. If failures occur during machine learning stage, partitions on faulty nodes will automatically be recomputed based on their lineage.

\section{Implementation}

While implementing Shark, we discovered that a number of engineering details had significant performance impacts. Overall, to improve the query processing speed, one should minimize the tail latency of tasks and the CPU cost of processing each row.

Memory-based Shuffle: Both Spark and Hadoop write map output files to disk, hoping that they will remain in the OS buffer cache when reduce tasks fetch them. In practice, we have found that the extra system calls and file system journaling adds significant overhead. In addition, the inability to control when buffer caches are flushed leads to variability in the execution time of shuffle tasks. A query's response time is determined by the last task to finish, and thus the increasing variability leads to long-tail latency, which significantly hurts shuffle performance. We modified the shuffle phase to materialize map outputs in memory, with the option to spill them to disk.

Temporary Object Creation: It is easy to write a program that creates many temporary objects, which can burden the JVM's garbage collector. For a parallel job, a slow GC at one task may slow the entire job. Shark operators and RDD transformations are written in a way that minimizes temporary object creations.

Bytecode Compilation of Expression Evaluators: In its current implementation, Shark sends the expression evaluators generated by the Hive parser as part of the tasks to be executed on each row. By profiling Shark, we discovered that for certain queries, when 
data is served out of the memory store the majority of the CPU cycles are wasted in interpreting these evaluators. We are working on a compiler to transform these expression evaluators into JVM bytecode, which can further increase the execution engine's throughput.

Specialized Data Structures: Using specialized data structures is another low-hanging optimization that we have yet to exploit. For example, Java's hash table is built for generic objects. When the hash key is a primitive type, the use of specialized data structures can lead to more compact data representations, and thus better cache behavior.

\section{Experiments}

We evaluated Shark using four datasets:

1. Pavlo et al. Benchmark: 2.1 TB of data reproducing Pavlo et al.'s comparison of MapReduce vs. analytical DBMSs [25].

2. TPC-H Dataset: $100 \mathrm{~GB}$ and $1 \mathrm{~TB}$ datasets generated by the DBGEN program [29].

3. Real Hive Warehouse: 1.7 TB of sampled Hive warehouse data from an early industrial user of Shark.

4. Machine Learning Dataset: $100 \mathrm{~GB}$ synthetic dataset to measure the performance of machine learning algorithms.

Overall, our results show that Shark can perform up to $100 \times$ faster than Hive, even though we have yet to implement some of the performance optimizations mentioned in the previous section. In particular, Shark provides comparable performance gains to those reported for MPP databases in Pavlo et al.'s comparison [25]. In some cases where data fits in memory, Shark exceeds the performance reported for MPP databases.

We emphasize that we are not claiming that Shark is fundamentally faster than MPP databases; there is no reason why MPP engines could not implement the same processing optimizations as Shark. Indeed, our implementation has several disadvantages relative to commercial engines, such as running on the JVM. Instead, we aim to show that it is possible to achieve comparable performance while retaining a MapReduce-like engine, and the finegrained fault recovery features that such engines provide. In addition, Shark can leverage this engine to perform high-speed machine learning functions on the same data, which we believe will be essential in future analytics workloads.

\subsection{Methodology and Cluster Setup}

Unless otherwise specified, experiments were conducted on Amazon EC2 using $100 \mathrm{~m} 2.4 \mathrm{xl}$ arge nodes. Each node had 8 virtual cores, $68 \mathrm{~GB}$ of memory, and 1.6 TB of local storage.

The cluster was running 64-bit Linux 3.2.28, Apache Hadoop 0.20.205, and Apache Hive 0.9. For Hadoop MapReduce, the number of map tasks and the number of reduce tasks per node were set to 8 , matching the number of cores. For Hive, we enabled JVM reuse between tasks and avoided merging small output files, which would take an extra step after each query to perform the merge.

We executed each query six times, discarded the first run, and report the average of the remaining five runs. We discard the first run in order to allow the JVM's just-in-time compiler to optimize common code paths. We believe that this more closely mirrors realworld deployments where the JVM will be reused by many queries.

\subsection{Pavlo et al. Benchmarks}

Pavlo et al. compared Hadoop versus MPP databases and showed that Hadoop excelled at data ingress, but performed unfavorably in query execution [25]. We reused the dataset and queries from their benchmarks to compare Shark against Hive.

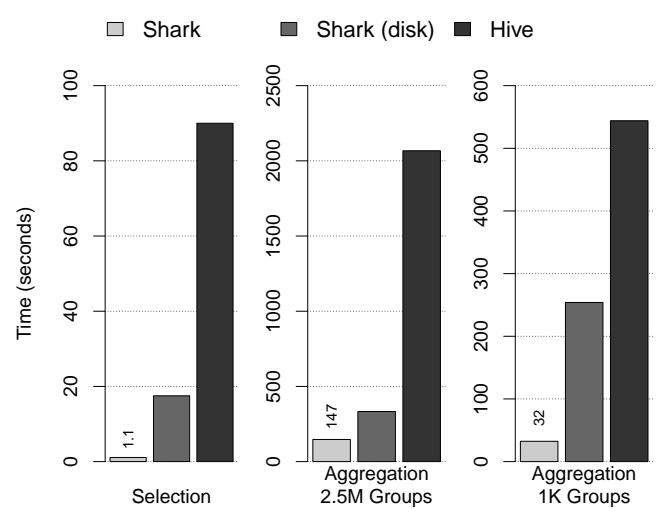

Figure 5: Selection and aggregation query runtimes (seconds) from Pavlo et al. benchmark

The benchmark used two tables: a $1 \mathrm{~GB} /$ node rankings table, and a $20 \mathrm{~GB} /$ node uservisits table. For our 100-node cluster, we recreated a $100 \mathrm{~GB}$ rankings table containing 1.8 billion rows and a 2 TB uservisits table containing 15.5 billion rows. We ran the four queries in their experiments comparing Shark with Hive and report the results in Figures 5 and 6 . In this subsection, we handtuned Hive's number of reduce tasks to produce optimal results for Hive. Despite this tuning, Shark outperformed Hive in all cases by a wide margin.

\subsubsection{Selection Query}

The first query was a simple selection on the rankings table:

SELECT pageURL, pageRank

FROM rankings WHERE pageRank > X;

In [25], Vertica outperformed Hadoop by a factor of 10 because a clustered index was created for Vertica. Even without a clustered index, Shark was able to execute this query $80 \times$ faster than Hive for in-memory data, and $5 \times$ on data read from HDFS.

\subsubsection{Aggregation Queries}

The Pavlo et al. benchmark ran two aggregation queries:

SELECT sourCeIP, SUM (adRevenue)

FROM uservisits GROUP BY sourceIP;

SELECT SUBSTR (sourceIP, 1, 7), SUM (adRevenue) FROM uservisits GROUP BY SUBSTR (sourceIP, 1, 7);

In our dataset, the first query had two million groups and the second had approximately one thousand groups. Shark and Hive both applied task-local aggregations and shuffled the data to parallelize the final merge aggregation. Again, Shark outperformed Hive by a wide margin. The benchmarked MPP databases perform local aggregations on each node, and then send all aggregates to a single query coordinator for the final merging; this performed very well when the number of groups was small, but performed worse with large number of groups. The MPP databases' chosen plan is similar to choosing a single reduce task for Shark and Hive.

\subsubsection{Join Query}

The final query from Pavlo et al. involved joining the 2 TB uservisits table with the $100 \mathrm{~GB}$ rankings table.

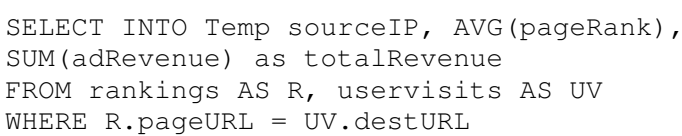




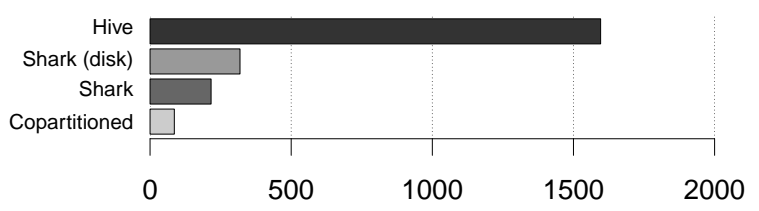

Figure 6: Join query runtime (seconds) from Pavlo benchmark

AND UV.visitDate BETWEEN Date ('2000-01-15')

AND Date ('2000-01-22')

GROUP BY UV.sourceIP;

Again, Shark outperformed Hive in all cases. Figure 6 shows that for this query, serving data out of memory did not provide much benefit over disk. This is because the cost of the join step dominated the query processing. Co-partitioning the two tables, however, provided significant benefits as it avoided shuffling data $2.1 \mathrm{~TB}$ of data during the join step.

\subsubsection{Data Loading}

Hadoop was shown by [25] to excel at data loading, as its data loading throughput was five to ten times higher than that of MPP databases. As explained in Section 2, Shark can be used to query data in HDFS directly, which means its data ingress rate is at least as fast as Hadoop's.

After generating the $2 \mathrm{~TB}$ uservisits table, we measured the time to load it into HDFS and compared that with the time to load it into Shark's memory store. We found the rate of data ingress was $5 \times$ higher in Shark's memory store than that of HDFS.

\subsection{Micro-Benchmarks}

To understand the factors affecting Shark's performance, we conducted a sequence of micro-benchmarks. We generated $100 \mathrm{~GB}$ and $1 \mathrm{~TB}$ of data using the DBGEN program provided by TPC$\mathrm{H}$ [29]. We chose this dataset because it contains tables and columns of varying cardinality and can be used to create a myriad of microbenchmarks for testing individual operators.

While performing experiments, we found that Hive and Hadoop MapReduce were very sensitive to the number of reducers set for a job. Hive's optimizer automatically sets the number of reducers based on the estimated data size. However, we found that Hive's optimizer frequently made the wrong decision, leading to incredibly long query execution times. We hand-tuned the number of reducers for Hive based on characteristics of the queries and through trial and error. We report Hive performance numbers for both optimizerdetermined and hand-tuned numbers of reducers. Shark, on the other hand, was much less sensitive to the number of reducers and required minimal tuning.

\subsubsection{Aggregation Performance}

We tested the performance of aggregations by running group-by queries on the TPH-H lineitem table. For the 100 GB dataset, lineitem table contained 600 million rows. For the 1 TB dataset, it contained 6 billion rows.

The queries were of the form:

SELECT [GROUP_BY_COLUMN], COUNT(*) FROM lineitem GROUP BY [GROUP_BY_COLUMN]

We chose to run one query with no group-by column (i.e., a simple count), and three queries with group-by aggregations: SHIPMODE (7 groups), RECEIPTDATE (2500 groups), and SHIPMODE (150 million groups in $100 \mathrm{~GB}$, and 537 million groups in $1 \mathrm{~TB}$ ).

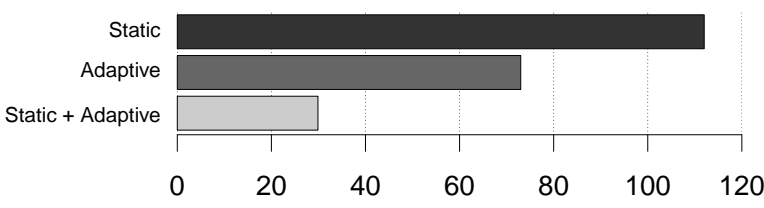

Figure 8: Join strategies chosen by optimizers (seconds)

For both Shark and Hive, aggregations were first performed on each partition, and then the intermediate aggregated results were partitioned and sent to reduce tasks to produce the final aggregation. As the number of groups becomes larger, more data needs to be shuffled across the network.

Figure 7 compares the performance of Shark and Hive, measuring Shark's performance on both in-memory data and data loaded from HDFS. As can be seen in the figure, Shark was $80 \times$ faster than hand-tuned Hive for queries with small numbers of groups, and $20 \times$ faster for queries with large numbers of groups, where the shuffle phase domniated the total execution cost.

We were somewhat surprised by the performance gain observed for on-disk data in Shark. After all, both Shark and Hive had to read data from HDFS and deserialize it for query processing. This difference, however, can be explained by Shark's very low task launching overhead, optimized shuffle operator, and other factors; see Section 7 for more details.

\subsubsection{Join Selection at Run-time}

In this experiment, we tested how partial DAG execution can improve query performance through run-time re-optimization of query plans. The query joined the lineitem and supplier tables from the 1 TB TPC-H dataset, using a UDF to select suppliers of interest based on their addresses. In this specific instance, the UDF selected 1000 out of 10 million suppliers. Figure 8 summarizes these results.

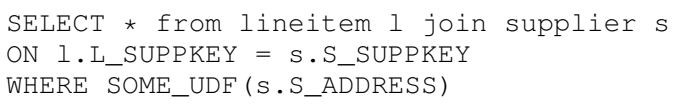

Lacking good selectivity estimation on the UDF, a static optimizer would choose to perform a shuffle join on these two tables because the initial sizes of both tables are large. Leveraging partial DAG execution, after running the pre-shuffle map stages for both tables, Shark's dynamic optimizer realized that the filtered supplier table was small. It decided to perform a map-join, replicating the filtered supplier table to all nodes and performing the join using only map tasks on lineitem.

To further improve the execution, the optimizer can analyze the logical plan and infer that the probability of supplier table being small is much higher than that of lineitem (since supplier is smaller initially, and there is a filter predicate on supplier). The optimizer chose to pre-shuffle only the supplier table, and avoided launching two waves of tasks on lineitem. This combination of static query analysis and partial DAG execution led to a $3 \times$ performance improvement over a naïve, statically chosen plan.

\subsubsection{Fault Tolerance}

To measure Shark's performance in the presence of node failures, we simulated failures and measured query performance before, during, and after failure recovery. Figure 9 summarizes fives runs of our failure recovery experiment, which was performed on a 50node $\mathrm{m} 2.4 \mathrm{xl}$ arge EC2 cluster.

We used a group-by query on the $100 \mathrm{~GB}$ lineitem table to measure query performance in the presence of faults. After loading the 

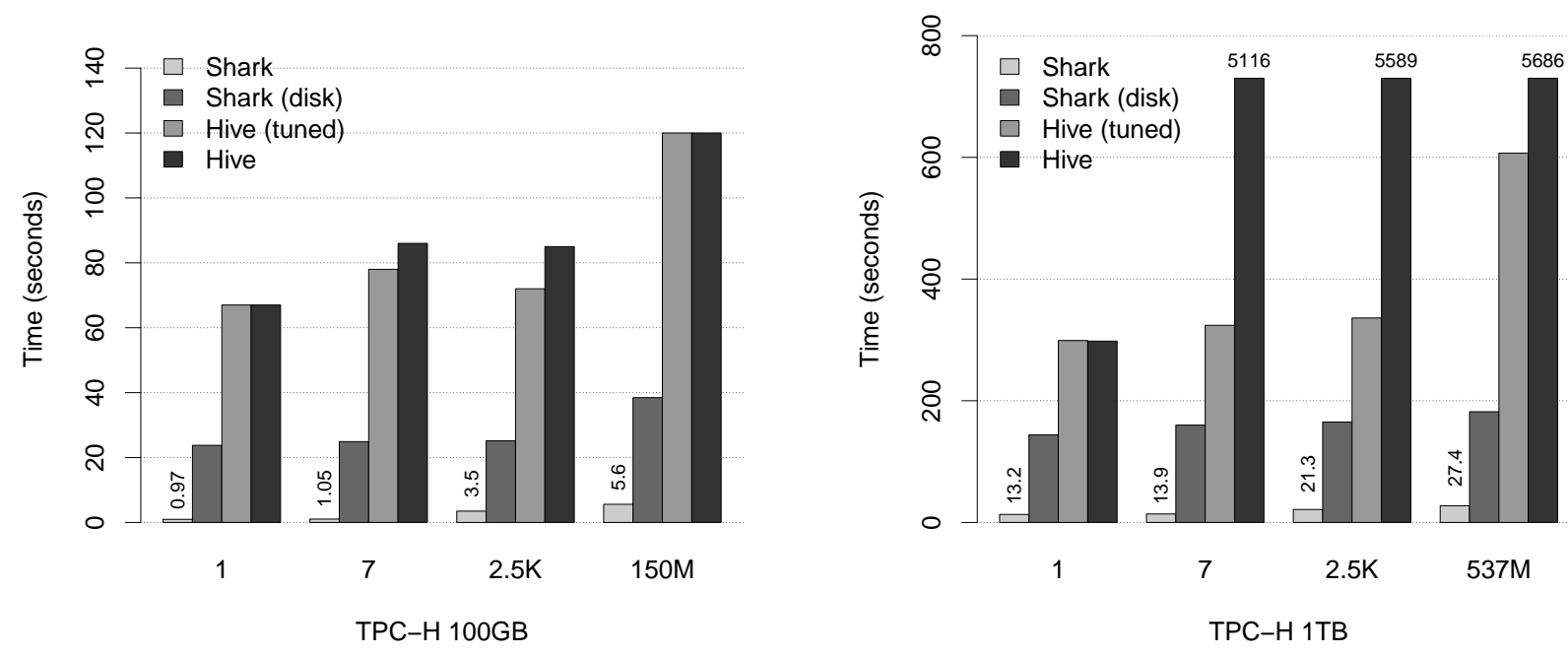

Figure 7: Aggregation queries on lineitem table. $\mathrm{X}$-axis indicates the number of groups for each aggregation query.

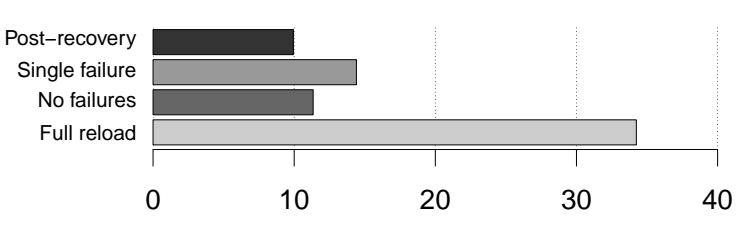

Figure 9: Query time with failures (seconds)

lineitem data into Shark's memory store, we killed a worker machine and re-ran the query. Shark gracefully recovered from this failure and parallelized the reconstruction of lost partitions on the other 49 nodes. This recovery had a small performance impact $(\sim 3$ seconds), but it was significantly cheaper than the cost of re-loading the entire dataset and re-executing the query.

After this recovery, subsequent queries operated against the recovered dataset, albeit with fewer machines. In Figure 9, the postrecovery performance was marginally better than the pre-failure performance; we believe that this was a side-effect of the JVM's JIT compiler, as more of the scheduler's code might have become compiled by the time the post-recovery queries were run.

\subsection{Real Hive Warehouse Queries}

An early industrial user provided us with a sample of their Hive warehouse data and two years of query traces from their Hive system. A leading video analytics company for content providers and publishers, the user built most of their analytics stack based on Hadoop. The sample we obtained contained 30 days of video session data, occupying $1.7 \mathrm{~TB}$ of disk space when decompressed. It consists of a single fact table containing 103 columns, with heavy use of complex data types such as array and struct. The sampled query log contains 3833 analytical queries, sorted in order of frequency. We filtered out queries that invoked proprietary UDFs and picked four frequent queries that are prototypical of other queries in the complete trace. These queries compute aggregate video quality metrics over different audience segments:

1. Query 1 computes summary statistics in 12 dimensions for users of a specific customer on a specific day.

2. Query 2 counts the number of sessions and distinct customer/client combination grouped by countries with filter predi-

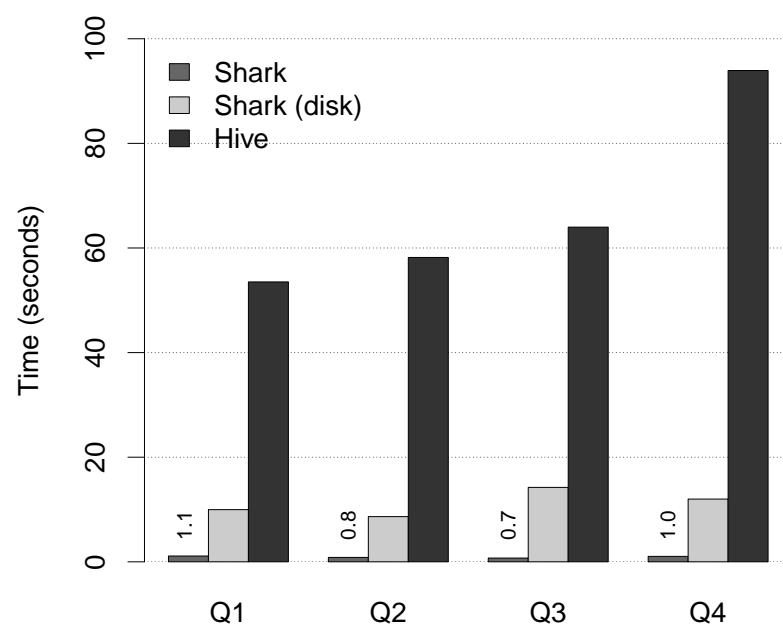

Figure 10: Real Hive warehouse workloads

cates on eight columns.

3. Query 3 counts the number of sessions and distinct users for all but 2 countries.

4. Query 4 computes summary statistics in 7 dimensions grouping by a column, and showing the top groups sorted in descending order.

Figure 10 compares the performance of Shark and Hive on these queries. The result is very promising as Shark was able to process these real life queries in sub-second latency in all but one cases, whereas it took Hive 50 to 100 times longer to execute them.

A closer look into these queries suggests that this data exhibits the natural clustering properties mentioned in Section 3.5. The map pruning technique, on average, reduced the amount of data scanned by a factor of 30 .

\subsection{Machine Learning}

A key motivator of using SQL in a MapReduce environment is the ability to perform sophisticated machine learning on big data. We implemented two iterative machine learning algorithms, logistic regression and k-means, to compare the performance of Shark versus 


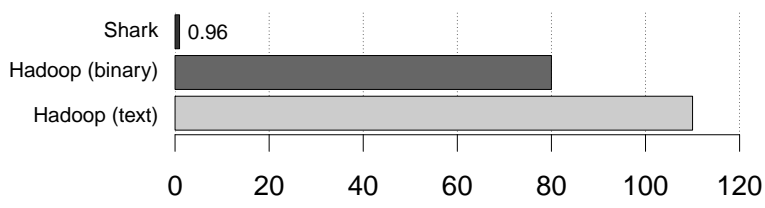

Figure 11: Logistic regression, per-iteration runtime (seconds)

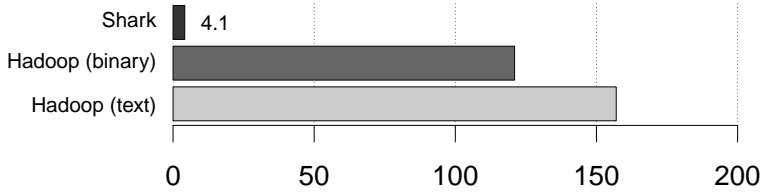

Figure 12: K-means clustering, per-iteration runtime (seconds)

running the same workflow in Hive and Hadoop.

The dataset was synthetically generated and contained 1 billion rows and 10 columns, occupying $100 \mathrm{~GB}$ of space. Thus, the feature matrix contained 1 billion points, each with 10 dimensions. These machine learning experiments were performed on a 100node m1 . xlarge EC2 cluster.

Data was initially stored in relational form in Shark's memory store and HDFS. The workflow consisted of three steps: (1) selecting the data of interest from the warehouse using SQL, (2) extracting features, and (3) applying iterative algorithms. In step 3, both algorithms were run for 10 iterations.

Figures 11 and 12 show the time to execute a single iteration of logistic regression and k-means, respectively. We implemented two versions of the algorithms for Hadoop, one storing input data as text in HDFS and the other using a serialized binary format. The binary representation was more compact and had lower CPU cost in record deserialization, leading to improved performance. Our results show that Shark is $100 \times$ faster than Hive and Hadoop for logistic regression and $30 \times$ faster for k-means. K-means experienced less speedup because it was computationally more expensive than logistic regression, thus making the workflow more CPU-bound.

In the case of Shark, if data initially resided in its memory store, step 1 and 2 were executed in roughly the same time it took to run one iteration of the machine learning algorithm. If data was not loaded into the memory store, the first iteration took 40 seconds for both algorithms. Subsequent iterations, however, reported numbers consistent with Figures 11 and 12. In the case of Hive and Hadoop, every iteration took the reported time because data was loaded from HDFS for every iteration.

\section{Discussion}

Shark shows that it is possible to run fast relational queries in a fault-tolerant manner using the fine-grained deterministic task model introduced by MapReduce. This design offers an effective way to scale query processing to ever-larger workloads, and to combine it with rich analytics. In this section, we consider two questions: first, why were previous MapReduce-based systems, such as Hive, slow, and what gave Shark its advantages? Second, are there other benefits to the fine-grained task model? We argue that fine-grained tasks also help with multitenancy and elasticity, as has been demonstrated in MapReduce systems.

\subsection{Why are Previous MapReduce-Based Systems Slow?}

Conventional wisdom is that MapReduce is slower than MPP databases for several reasons: expensive data materialization for fault toler- ance, inferior data layout (e.g., lack of indices), and costlier execution strategies $[25,26]$. Our exploration of Hive confirms these reasons, but also shows that a combination of conceptually simple "engineering" changes to the engine (e.g., in-memory storage) and more involved architectural changes (e.g., partial DAG execution) can alleviate them. We also find that a somewhat surprising variable not considered in detail in MapReduce systems, the task scheduling overhead, actually has a dramatic effect on performance, and greatly improves load balancing if minimized.

Intermediate Outputs: MapReduce-based query engines, such as Hive, materialize intermediate data to disk in two situations. First, within a MapReduce job, the map tasks save their output in case a reduce task fails [13]. Second, many queries need to be compiled into multiple MapReduce steps, and engines rely on replicated file systems, such as HDFS, to store the output of each step.

For the first case, we note that map outputs were stored on disk primarily as a convenience to ensure there is sufficient space to hold them in large batch jobs. Map outputs are not replicated across nodes, so they will still be lost if the mapper node fails [13]. Thus, if the outputs fit in memory, it makes sense to store them in memory initially, and only spill them to disk if they are large. Shark's shuffle implementation does this by default, and sees far faster shuffle performance (and no seeks) when the outputs fit in RAM. This is often the case in aggregations and filtering queries that return a much smaller output than their input. ${ }^{5}$ Another hardware trend that may improve performance, even for large shuffles, is SSDs, which would allow fast random access to a larger space than memory.

For the second case, engines that extend the MapReduce execution model to general task DAGs can run multi-stage jobs without materializing any outputs to HDFS. Many such engines have been proposed, including Dryad, Tenzing and Spark [17, 9, 33].

Data Format and Layout: While the naïve pure schema-on-read approach to MapReduce incurs considerable processing costs, many systems use more efficient storage formats within the MapReduce model to speed up queries. Hive itself supports "table partitions" (a basic index-like system where it knows that certain key ranges are contained in certain files, so it can avoid scanning a whole table), as well as column-oriented representation of on-disk data [28]. We go further in Shark by using fast in-memory columnar representations within Spark. Shark does this without modifying the Spark runtime by simply representing a block of tuples as a single Spark record (one Java object from Spark's perspective), and choosing its own representation for the tuples within this object.

Another feature of Spark that helps Shark, but was not present in previous MapReduce runtimes, is control over the data partitioning across nodes (Section 3.4). This lets us co-partition tables.

Finally, one capability of RDDs that we do not yet exploit is random reads. While RDDs only support coarse-grained operations for their writes, read operations on them can be fine-grained, accessing just one record [33]. This would allow RDDs to be used as indices. Tenzing can use such remote-lookup reads for joins [9].

Execution Strategies: Hive spends considerable time on sorting the data before each shuffle and writing the outputs of each MapReduce stage to HDFS, both limitations of the rigid, one-pass MapReduce model in Hadoop. More general runtime engines, such as Spark, alleviate some of these problems. For instance, Spark supports hash-based distributed aggregation and general task DAGs.

\footnotetext{
${ }^{5}$ Systems like Hadoop also benefit from the OS buffer cache in serving map outputs, but we found that the extra system calls and file system journalling from writing map outputs to files still adds overhead (Section 5).
} 
To truly optimize the execution of relational queries, however, we found it necessary to select execution plans based on data statistics. This becomes difficult in the presence of UDFs and complex analytics functions, which we seek to support as first-class citizens in Shark. To address this problem, we proposed partial DAG execution (PDE), which allows our modified version of Spark to change the downstream portion of an execution graph once each stage completes based on data statistics. PDE goes beyond the runtime graph rewriting features in previous systems, such as DryadLINQ [19], by collecting fine-grained statistics about ranges of keys and by allowing switches to a completely different join strategy, such as broadcast join, instead of just selecting the number of reduce tasks.

Task Scheduling Cost: Perhaps the most surprising engine property that affected Shark, however, was a purely "engineering" concern: the overhead of launching tasks. Traditional MapReduce systems, such as Hadoop, were designed for multi-hour batch jobs consisting of tasks that were several minutes long. They launched each task in a separate OS process, and in some cases had a high latency to even submit a task. For instance, Hadoop uses periodic "heartbeats" from each worker every 3 seconds to assign tasks, and sees overall task startup delays of 5-10 seconds. This was sufficient for batch workloads, but clearly falls short for ad-hoc queries.

Spark avoids this problem by using a fast event-driven RPC library to launch tasks and by reusing its worker processes. It can launch thousands of tasks per second with only about $5 \mathrm{~ms}$ of overhead per task, making task lengths of 50-100 ms and MapReduce jobs of $500 \mathrm{~ms}$ viable. What surprised us is how much this affected query performance, even in large (multi-minute) queries.

Sub-second tasks allow the engine to balance work across nodes extremely well, even when some nodes incur unpredictable delays (e.g., network delays or JVM garbage collection). They also help dramatically with skew. Consider, for example, a system that needs to run a hash aggregation on 100 cores. If the system launches 100 reduce tasks, the key range for each task needs to be carefully chosen, as any imbalance will slow down the entire job. If it could split the work among 1000 tasks, then the slowest task can be as much as $10 \times$ slower than the average without affecting the job response time much! After implementing skew-aware partition selection in PDE, we were somewhat disappointed that it did not help compared to just having a higher number of reduce tasks in most workloads, because Spark could comfortably support thousands of such tasks. However, this property makes the engine highly robust to unexpected skew.

In this way, Spark stands in contrast to Hadoop/Hive, where using the wrong number of tasks was sometimes $10 \times$ slower than an optimal plan, and there has been considerable work to automatically choose the number of reduce tasks [21, 15]. Figure 13 shows how job execution times varies as the number of reduce tasks launched by Hadoop and Spark. Since a Spark job can launch thousands of reduce tasks without incurring much overhead, partition data skew can be mitigated by always launching many tasks.
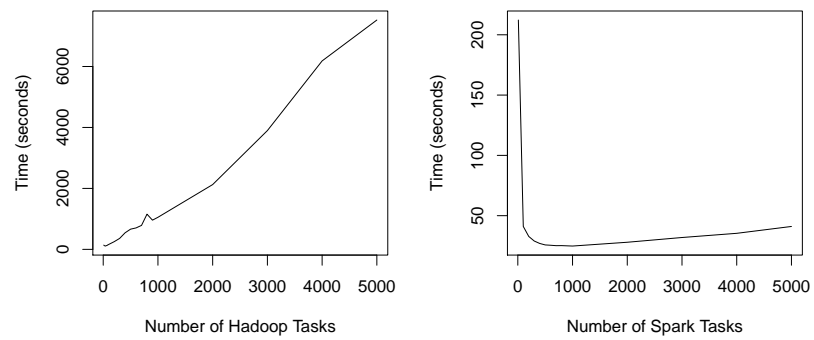

Figure 13: Task launching overhead
More fundamentally, there are few reasons why sub-second tasks should not be feasible even at higher scales than we have explored, such as tens of thousands of nodes. Systems like Dremel [24] routinely run sub-second, multi-thousand-node jobs. Indeed, even if a single master cannot keep up with the scheduling decisions, the scheduling could be delegated across "lieutenant" masters for subsets of the cluster. Fine-grained tasks also offer many advantages over coarser-grained execution graphs beyond load balancing, such as faster recovery (by spreading out lost tasks across more nodes) and query elasticity; we discuss some of these next.

\subsection{Other Benefits of the Fine-Grained Task Model}

While this paper has focused primarily on the fault tolerance benefits of fine-grained deterministic tasks, the model also provides other attractive properties. We wish to point out two benefits that have been explored in MapReduce-based systems.

Elasticity: In traditional MPP databases, a distributed query plan is selected once, and the system needs to run at that level of parallelism for the whole duration of the query. In a fine-grained task system, however, nodes can appear or go away during a query, and pending work will automatically be spread onto them. This enables the database engine to naturally be elastic. If an administrator wishes to remove nodes from the engine (e.g., in a virtualized corporate data center), the engine can simply treat those as failed, or (better yet) proactively replicate their data to other nodes if given a few minutes' warning. Similarly, a database engine running on a cloud could scale $u p$ by requesting new VMs if a query is expensive. Amazon's Elastic MapReduce [2] already supports resizing clusters at runtime.

Multitenancy: The same elasticity, mentioned above, enables dynamic resource sharing between users. In a traditional MPP database, if an important query arrives while another large query using most of the cluster, there are few options beyond canceling the earlier query. In systems based on fine-grained tasks, one can simply wait a few seconds for the current tasks from the first query to finish, and start giving the nodes tasks from the second query. For instance, Facebook and Microsoft have developed fair schedulers for Hadoop and Dryad that allow large historical queries, computeintensive machine learning jobs, and short ad-hoc queries to safely coexist $[32,18]$.

\section{Related Work}

To the best of our knowledge, Shark is the only low-latency system that can efficiently combine SQL and machine learning workloads, while supporting fine-grained fault recovery.

We categorize large-scale data analytics systems into three classes. First, systems like Hive [28], Tenzing [9], SCOPE [8], and Cheetah [10] compile declarative queries into MapReduce-style jobs. Even though some of them introduce modifications to the execution engine they are built on, it is hard for these systems to achieve interactive query response times for reasons discussed in Section 7.

Second, several projects aim to provide low-latency engines using architectures resembling shared-nothing parallel databases. Such projects include PowerDrill [16] and Impala [1]. These systems do not support fine-grained fault tolerance. In case of mid-query faults, the entire query needs to be re-executed. Google's Dremel [24] does rerun lost tasks, but it only supports an aggregation tree topology for query execution, and not the more complex shuffle DAGs required for large joins or distributed machine learning.

A third class of systems take a hybrid approach by combining a MapReduce-like engine with relational databases. HadoopDB [3] connects multiple single-node database systems using Hadoop as 
the communication layer. Queries can be parallelized using Hadoop MapReduce, but within each MapReduce task, data processing is pushed into the relational database system. Osprey [31] is a middleware layer that adds fault-tolerance properties to parallel databases. It does so by breaking a SQL query into multiple small queries and sending them to parallel databases for execution. Shark presents a much simpler single-system architecture that supports all of the properties of this third class of systems, as well as statistical learning capabilities that HadoopDB and Osprey lack.

The partial DAG execution (PDE) technique introduced by Shark resembles adaptive query optimization techniques proposed in [6, $30,20]$. It is, however, unclear how these single-node techniques would work in a distributed setting and scale out to hundreds of nodes. In fact, PDE actually complements some of these techniques, as Shark can use PDE to optimize how data gets shuffled across nodes, and use the traditional single-node techniques within a local task. DryadLINQ [19] optimizes its number of reduce tasks at run-time based on map output sizes, but does not collect richer statistics, such as histograms, or make broader execution plan changes, such as changing join algorithms, like PDE can. RoPE [4] proposes using historical query information to optimize query plans, but relies on repeatedly executed queries. PDE works on queries that are executing for the first time.

Finally, Shark builds on the distributed approaches for machine learning developed in systems like Graphlab [22], Haloop [7], and Spark [33]. However, Shark is unique in offering these capabilities in a SQL engine, allowing users to select data of interest using SQL and immediately run learning algorithms on it without timeconsuming export to another system. Compared to Spark, Shark also provides far more efficient in-memory representation of relational data, and mid-query optimization using PDE.

\section{Conclusion}

We have presented Shark, a new data warehouse system that combines fast relational queries and complex analytics in a single, faulttolerant runtime. Shark generalizes a MapReduce-like runtime to run SQL effectively, using both traditional database techniques, such as column-oriented storage, and a novel partial DAG execution $(P D E)$ technique that lets it reoptimize queries at run-time based on fine-grained data statistics. This designs enables Shark to generally match the speedups reported for MPP databases over MapReduce, while simultaneously providing machine learning functions in the same engine and fine-grained, mid-query fault tolerance across both SQL and machine learning. Overall, the system is up to $100 \times$ faster than Hive for SQL, and $100 \times$ faster than Hadoop for machine learning.

We have open sourced Shark at shark. cs.berkeley . edu, and have also worked with two Internet companies as early users. They report speedups of $40-100 \times$ on real queries, consistent with our results.

\section{Acknowledgments}

We thank Cliff Engle, Harvey Feng, Shivaram Venkataraman, Ram Sriharsha, Denny Britz, Antonio Lupher, Patrick Wendell, and Paul Ruan for their work on Shark. This research is supported in part by NSF CISE Expeditions award CCF-1139158, gifts from Amazon Web Services, Google, SAP, Blue Goji, Cisco, Cloudera, Ericsson, General Electric, Hewlett Packard, Huawei, Intel, Microsoft, NetApp, Oracle, Quanta, Splunk, VMware and by DARPA (contract \#FA8650-11-C-7136).

\section{References}

[1] https://github.com/cloudera/impala.

[2] http://aws.amazon.com/about-aws/whats-new/2010/10/20/amazonelastic-mapreduce-introduces-resizing-running-job-flows/.

[3] A. Abouzeid et al. Hadoopdb: an architectural hybrid of mapreduce and dbms technologies for analytical workloads. VLDB, 2009.

[4] S. Agarwal et al. Re-optimizing data-parallel computing. In NSDI'12.

[5] G. Ananthanarayanan et al. Pacman: Coordinated memory caching for parallel jobs. In NSDI, 2012.

[6] R. Avnur and J. M. Hellerstein. Eddies: continuously adaptive query processing. In SIGMOD, 2000.

[7] Y. Bu et al. HaLoop: efficient iterative data processing on large clusters. Proc. VLDB Endow., 2010.

[8] R. Chaiken et al. Scope: easy and efficient parallel processing of massive data sets. $V L D B, 2008$.

[9] B. Chattopadhyay, et al. Tenzing a sql implementation on the mapreduce framework. PVLDB, 4(12):1318-1327, 2011.

[10] S. Chen. Cheetah: a high performance, custom data warehouse on top of mapreduce. $V L D B, 2010$.

[11] C. Chu et al. Map-reduce for machine learning on multicore. Advances in neural information processing systems, 19:281, 2007.

[12] J. Cohen, B. Dolan, M. Dunlap, J. Hellerstein, and C. Welton. Mad skills: new analysis practices for big data. VLDB, 2009.

[13] J. Dean and S. Ghemawat. MapReduce: Simplified data processing on large clusters. In $O S D I, 2004$.

[14] X. Feng et al. Towards a unified architecture for in-rdbms analytics. In SIGMOD, 2012.

[15] B. Guffler et al. Handling data skew in mapreduce. In CLOSER, 2011.

[16] A. Hall et al. Processing a trillion cells per mouse click. VLDB.

[17] M. Isard et al. Dryad: distributed data-parallel programs from sequential building blocks. SIGOPS, 2007.

[18] M. Isard et al. Quincy: Fair scheduling for distributed computing clusters. In SOSP '09, 2009.

[19] M. Isard and Y. Yu. Distributed data-parallel computing using a high-level programming language. In SIGMOD, 2009.

[20] N. Kabra and D. J. DeWitt. Efficient mid-query re-optimization of sub-optimal query execution plans. In SIGMOD, 1998.

[21] Y. Kwon et al. Skewtune: mitigating skew in mapreduce applications. In SIGMOD '12, 2012.

[22] Y. Low et al. Distributed graphlab: a framework for machine learning and data mining in the cloud. $V L D B, 2012$.

[23] G. Malewicz et al. Pregel: a system for large-scale graph processing. In SIGMOD, 2010.

[24] S. Melnik et al. Dremel: interactive analysis of web-scale datasets. Proc. VLDB Endow., 3:330-339, Sept 2010.

[25] A. Pavlo et al. A comparison of approaches to large-scale data analysis. In SIGMOD, 2009.

[26] M. Stonebraker et al. Mapreduce and parallel dbmss: friends or foes? Commun. ACM.

[27] M. Stonebraker et al. C-store: a column-oriented dbms. In $V L D B$, 2005.

[28] A. Thusoo et al. Hive-a petabyte scale data warehouse using hadoop. In ICDE, 2010.

[29] Transaction Processing Performance Council. TPC BENCHMARK H.

[30] T. Urhan, M. J. Franklin, and L. Amsaleg. Cost-based query scrambling for initial delays. In SIGMOD, 1998.

[31] C. Yang et al. Osprey: Implementing mapreduce-style fault tolerance in a shared-nothing distributed database. In ICDE, 2010.

[32] M. Zaharia et al. Delay scheduling: A simple technique for achieving locality and fairness in cluster scheduling. In EuroSys 10, 2010.

[33] M. Zaharia et al. Resilient distributed datasets: a fault-tolerant abstraction for in-memory cluster computing. NSDI, 2012. 